\title{
Infectious Diarrhea, a Public Health Problem in Population
}

Deac Liana Monica MD PhD

Public Health Institute, Babes - Bolyai University, Cluj, Romania

Corresponding Author: Deac Liana Monica, Public Health Institute, Babes - Bolyai University, Cluj, Romania.

Received date: March 26, 2021; Accepted date: June 04, 2021; Published date: June 08,2021

Citation: Deac L Monica. (2021) Infectious Diarrhea, a Public Health Problem in Population. Biomedical Research and Clinical Reviews. 4(2); DOI: $10.31579 / 2692-9406 / 058$

Copyright: (c) 2021 Deac Liana Monica, This is an open-access article distributed under the terms of the Creative Commons Attribution License, which permits unrestricted use, distribution, and reproduction in any medium, provided the original author and source are credited

\begin{abstract}
:
Infectious etiology in acute diarrhea, referred several gastrointestinal diseases, Most are gastroenteritis associated with clinical signs and symptoms including: nausea, vomiting, abdominal pain and cramps, bloating, flatulence, fever, passage of bloody stools, tenesmus, and fecal urgency disorders. Diarrheal illness is a large worldwide public health problem, with substantial regional variation, in the prevalence of medical signs by being caused by specific pathogens. Infectious diarrheal diseases, are the second leading cause of morbidity and mortality worldwide and can cause real public health concerns. Such diarrhea was studied as a medical disorder, in a 3 years period, 2017 to 2020, in Transylvania a large region in Romania. It was found there, 3577 number of diarrheal diseases cases, appeared almost during July to August. The case incidence for the disease arrived at $65 \%$, in the entire summer season. The cases data were transmitted by 12 district Sanitary Polices, to the Public Health Center Cluj. Cases were almost diagnosed by the family doctors of the region, in more then $50 \%$. Few of this have need some days of hospitalization, because of several disease disorders, in which case it was used a specific adequate fluid and electrolyte replacement as key of the treatment, for managing diarrheal illnesses. Even so, 3 children under 5 years age died, because of severe complications. It was remarked so, organic failed who was estimate to be the cause of their deaths. Clinical and epidemiological evaluations were done and even defined the severity and type of this mentioned illnesses. The detected infectious etiology for the acute diarrheas, where determined in authorized Microbiology laboratories, where were identified: Shigella spp, Salmonella spp, Campylobacter spp, Yersinia spp, Rotavirus, Giardia. Most number of cases appeared in children, being suggestive in $63 \%$, followed by elderly or adult people in $17 \%$ each. Acute diarrheal illness had to be considered a major public health issue, against which some determined control efforts are needed. Public health surveillance of infectious acute diarrhea, includes obligatory done strategies for a correct infection control.
\end{abstract}

Key words: acute diarrhea; symptoms; etiology; health survey

\section{Introduction}

On every year, according to the World Health Organization (WHO) and UNICEF data, there are about 2 billion cases of diarrheal disease worldwide. 1.9 million Children younger than 5 years of age, mostly in developing countries, perish from diarrhea each year. In industrialized countries, relatively few patients die from diarrhea, but even so, the disease continues to be an important cause of morbidity associated with substantial health care costs. However in the world, the morbidity from diarrheal diseases has remained relatively constant during the past 2 decades [4].

Because of all this, it was studied in the Epidemiology Department, from the Public Health Institute, during 2017 - 2020, the morbidity caused by infectious diarrhea, appeared in Transylvania region from Romania. Despite the economic and societal burdens of diarrheal illnesses, there are still few clinical guidelines, which exist in correct use for the diagnosis and treatment of persons with such suspected or relevant infectious diarrheas. That for, considerable clinical practice have been observed for to demonstrate a clear need of a clinical diagnostic, improved by specific guidelines.

\section{Material and Method}

An analytical descriptive epidemiological study, was used to analyze the 3577 cases of infectious diarrheas, in the population from Transylvania region in Romania. It was figured out all representative aspects of the pathology at: children, adults and elderly people. Data recruitment were ordered by the existing pathology in Ro National Public Health Guidelines. Obtained data transmitted by the territory sanitary police units, were analyzed on each year, during 5 months from May to November. In all the studied years, 2017-2020 this were sent to the Epidemiology Department in the Public Health Center Cluj, where it was realized an epidemiologic analyze and data interpretations

\section{RESULTS and COMMENTS}

Figure 1: Present the 3577 infectious diarrheas appeared during 2017 to 2020 in Transylvania region from Romania 


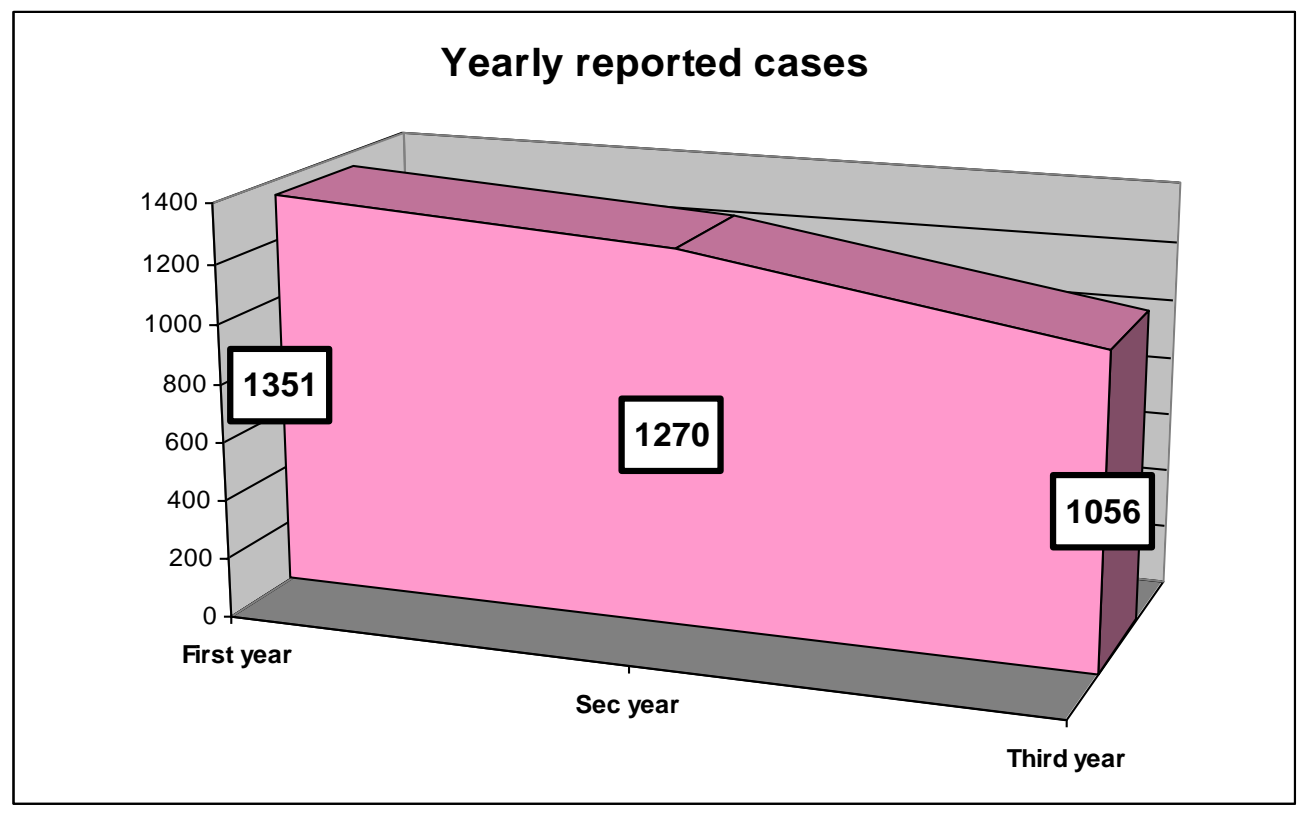

Figure 1: Number of Infectious diarrheas

Most of this disease were present in the first year of the study, in 37\%. In the second year this were followed by $35,5 \%$ and at least in the third study year, in $29,5 \%$. In all this 3 years, the infectious pathology appeared in the summer season, almost during July and August. In the studied territory it was not a significant estimate difference. The case incidence was $65 \%$, for the sum

Significant to be figured out is here, that infectious diarrhea, existed in the study, at each age decade. More significant it appeared to be in children, with $63 \%$, as Fig. 2 present data.

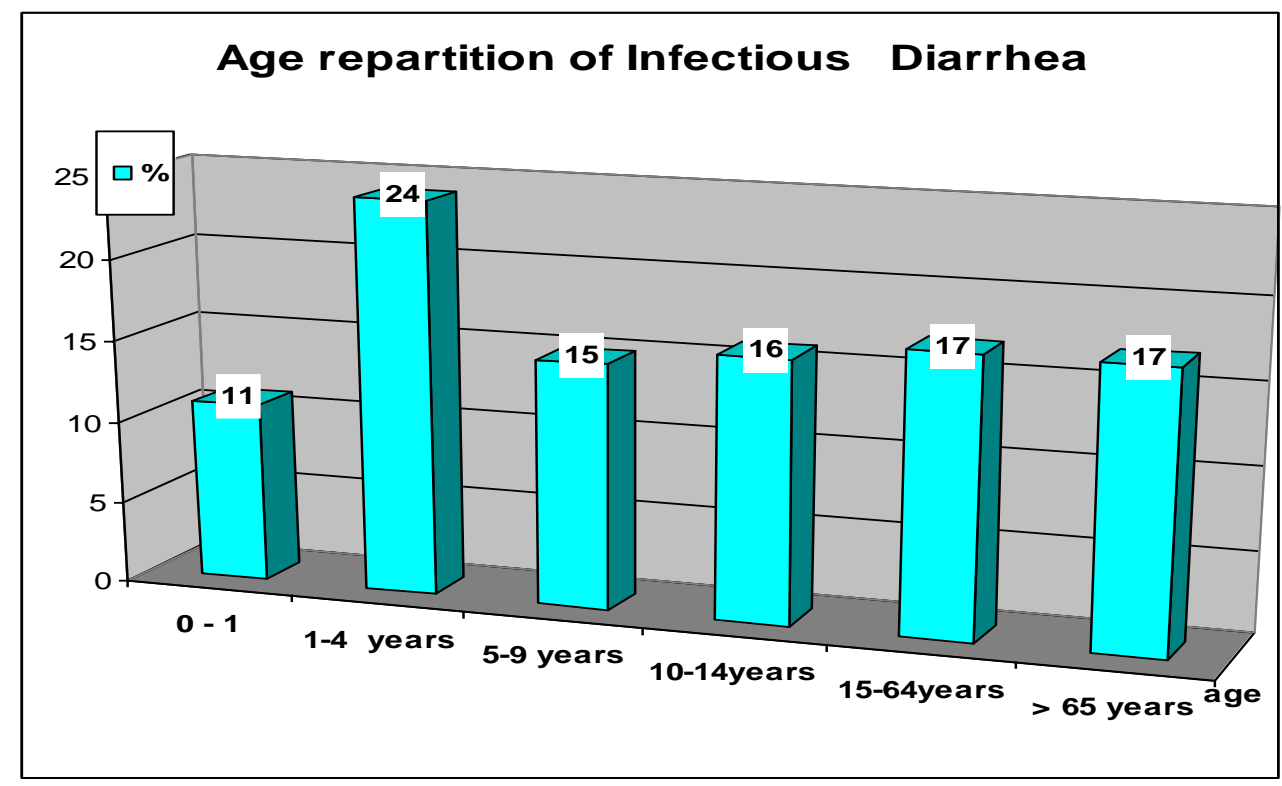

Figure 2: Age of infectious diarrheas

Data made relevant that the disease was present at children, at 0-14 age. In adults infectious diarrhea was remarked in $17 \%$, as well in elderly people this pathology was represented in same order of cases in $17 \% .27 \%$ of children and adults needed short hospitalization days, because of dehydration. For the disorders it was used a specific adequate fluid and electrolyte replacement, as key of the treatment and managing diarrheal illnesses. 3 children under 5 years age died, even after this specific therapy, because of severe organic complications. Organic fails caused their deaths.

Public international data, present o a growing awareness of the potentially with huge impact, in the developing world, of long-term disability, caused by repeated early childhood enteric infections. Worldwide, there are 3.1 million deaths due to diarrhea per year (18400 per day), mostly at young children in developing areas [1]. Annual deaths due to diarrhea globally occur mainly in young children, and the number of deaths is 1000 -fold 
higher than in the United States, where most of those who die of diarrheal illness, are elderly people [2].

Always, clinical and epidemiological evaluation must define the severity and type of this illnesses. A collaborative activity and work between clinicians and public health practitioners can make to get less severe evolution of the infectious diarrheas cases. That for clinicians, can early put the diagnosis of an acute episode of diarrhea and can lead to interventions that alleviate symptoms and prevent secondary possible other disease transmissions. For public health practitioners, prompt notification of pathogen-specific diagnoses and bacterial isolates through public health surveillance, can low the rates of transmission and lead to timely detection and control of any disease outbreaks. Because both clinicians and public health practitioners share overlapping responsibilities for such diagnosis, management, and prevention of infectious diarrheal diseases, must contain specific recommendations and specific guidelines for both groups of specialists [5].
Diarrheal illness is a problem worldwide, with substantial regional variation in the prevalence of specific pathogens. Testing of stool specimens is always indicated for each patient with enteric disorders. This had to be ordinate up to an existing national guideline, for to can identify all microbiological specimens. That for, it was figured out, the etiological aspects concerning all detected cases of infectious diarrheas. Data were received from several territory laboratories, where it was realized pertinent microbiological investigations. All this data were identified, in order to present the microorganisms and their aspects. With, it was possible to make an overview, concerning the heterogenic microorganisms, who made the appearance of the infectious diarrhea diseases, present in the Transylvania population study. The incidence of this aspects, is presented in Fig 3, in which it is shown out all identified germs.

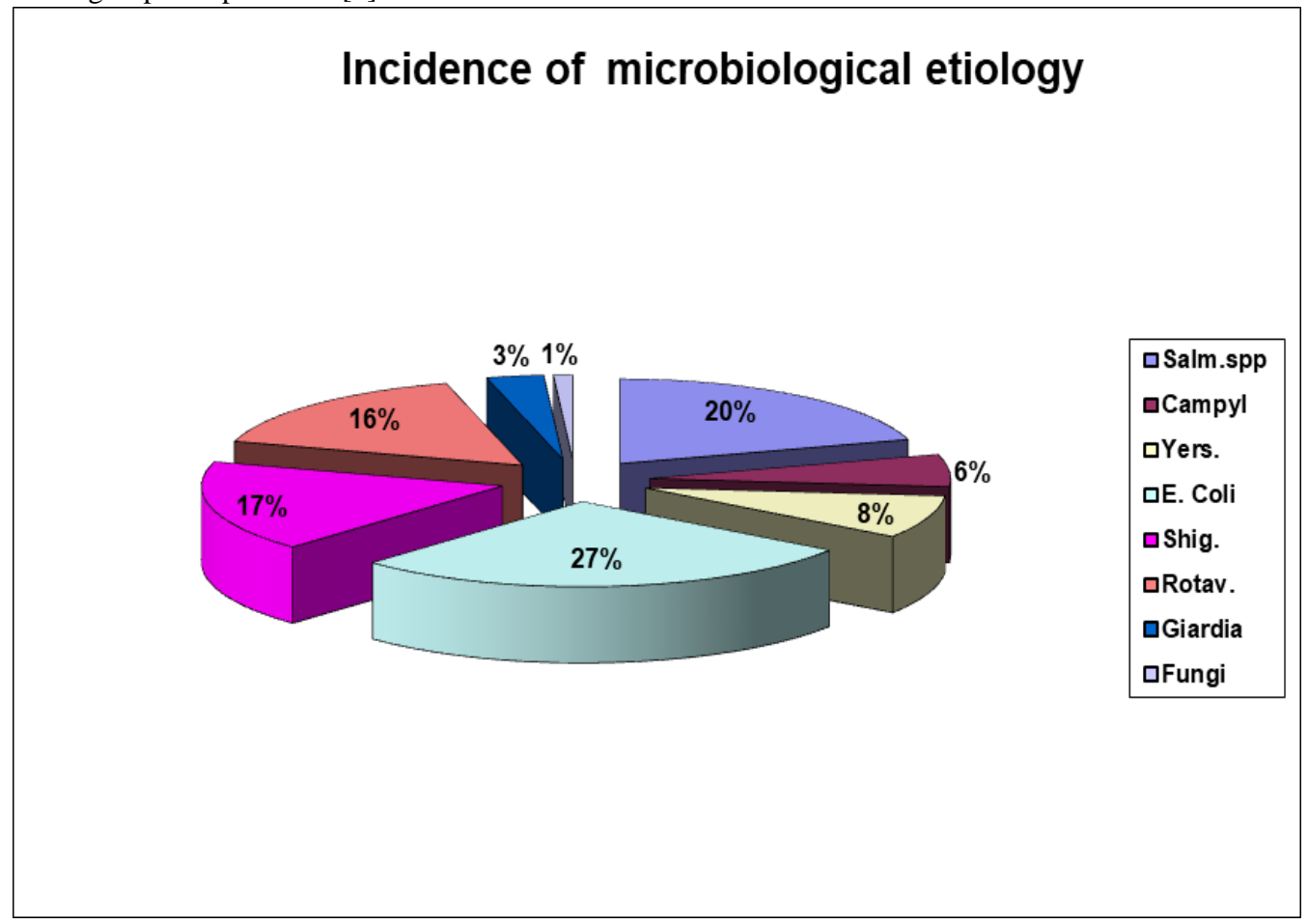

Figure 3: Detected microbiological aspects

Most of germs species belonged to the Enterobacteriaceae Family, represented by E coli in $27 \%$, Salmonella spp. in $20 \%$; Shigella spp., in $17 \%$. Important is to be figured out is, that the death of the 3 children under 5 age were caused by invasive E. coli, present in their stool samples. It is also important to be remarked the presence of Yersinia spp. in $8 \%$ and of Campylobacter spp... in $6 \%$, in other patients samples. The presence of the many different bacterial species were different identified: E. Coli in children faces and all other germs were present in adults, or in the elderly people samples. The observation mention the presence, in 17 $\%$ of Rotavirus, which mostly were present in the infectious diarrhea of the children. $3 \%$ of children have had even Giardia in their samples and $1 \%$ of elderly people have had Fungi in their preserved tools. Many of these organisms can easily be transmitted through infected food or water, or from person to person, as the epidemiological study figured out in the Transylvania territory infectious diarrhea cases.
Today, there are various interpretations of what is considered medically indicated for evaluating persons with infectious diarrhea. Stool cultures are often viewed as important tests, with a high result significances [3]. With the rapid globalization and industrialization of our food supply, more recognized pathogens can appear or can be figured out worldwide. More with a multiplicity of recognized pathogens, new Laboratory diagnostic of tool samples can be done and so can even change the known before aspects. The challenges of determining optimal, cost-effective means for such appropriate diagnosis, clinical management, and public health control of diarrheal illnesses knowledge [6]. This will continue to evolve as improved understanding of pathogenesis and development and can bring in use more rapid tests, who will improve the diagnosis and management of infectious diarrheal illness. It had to be accepted that this diseases are one of the most common clinical syndromes in our society. That's why the lack of a specific diagnosis today, can hinder appropriate management and treatment of many such infections. Observation of 
patients is a recommendation, for to provide always to clinicians and public health practitioners, consensus-based correct document. These will aid in the management of acute diarrheas, by addressing data, which patients to be tested, what tests to order, what medical treatments to be used, and what steps to take to ensure, that appropriate public health actions, are always implemented, well enough.

\section{Conclusions}

Any specific differences of date, could be offered, in the 3 year study and it was clear enough that infectious diarrhea appeared during the summer months, almost from July to August.

A significant incidence for infectious diarrhea appeared at Children, of 014 age group.

Each patient should be observed about potential epidemiological risk factors, for any diarrheal diseases, or for possible spread infection in the territory.

All bacterial, viral, and parasitic pathogens had to be identified, for evaluating all persons with any enteric infections.

To reduce the morbidity and possible mortality in infectious diarrheas, it is needed a serious clinical and public health study, that require specific control measures in clinics and health care activity group, for to identify optimal diagnostic, treatment, and prevention methods.

\section{References:}

1. Adkins HJ, Escamilla J, Santiago LT, et al. (1987) Two-year survey of etiologic agents of diarrheal disease at San Lazaro Hospital,Manila Republic of the Philippines. J Clin Microbiol. 25: 1143-1147.

2. Matthew R. Riley R. (2008) Dorsey Bass, in Pediatric Gastroenterology.

3. Guerrant RL, Van Gilder T, Steiner TS, et al. (2001) Infectious Diseases Society of America. Practice guidelines for the management of infectious diarrhea. Clin Infect Dis. 32(3):331351.

4. Snyder JD, Merson MH. (1982) The magnitude of the global problem of acute diarrheal disease: a review of active surveillance, data.Bull, WHO; 60:605-613.

5. WHO Program for the Control of Diarrheal Diseases. CD update on persistent diarrhea. No.4, March 1989.

6. WHO. The management and prevention of acute diarrhoea: practical guidelines. $3 \mathrm{~d}$ ed. Geneva: World Health Organization.

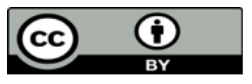

This work is licensed under Creative Commons Attribution 4.0 License

\section{To Submit Your Article Click Here: Submit Manuscript}

DOI: $10.31579 / 2692-9406 / 058$
Ready to submit your research? Choose Auctores and benefit from:

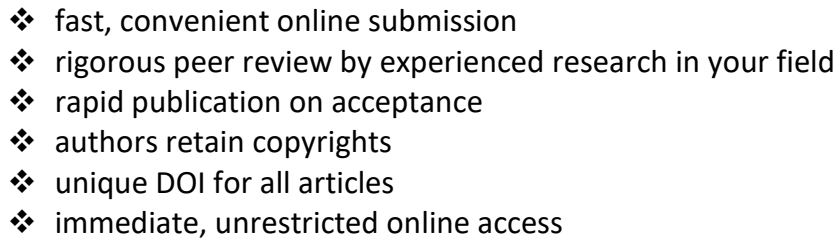

At Auctores, research is always in progress.

Learn more www.auctoresonline.org/journals/biomedical-research-andclinical-reviews-- 